\title{
EDITORIAL
}

\section{The Science of Nature}

\author{
Sven Thatje
}

Received: 5 March 2009 / Accepted: 5 March 2009/Published online: 18 March 2009

(C) Springer-Verlag 2009

Since its foundation in 1913, the body of academic work published in Naturwissenschaften (NAWI) has reflected contemporary developments across the breadth of the natural science disciplines. Achieving international acceptance as a multidisciplinary journal is challenging and potentially risky, but is ultimately a highly desirable goal, which only a few journals have been able to sustain over time. 2013 will mark 100 years of this journal's existence, and NAWI is now confirming its focus and future direction as one of the few multidisciplinary journals of present time.

Now more than ever, cross-boundary thinking is demanded of scientists of all disciplines, in order to tackle the challenges facing society, such as the global anthropogenic impact on our planet. Careers in the natural sciences of the past decades were largely driven by individual specialist development, often requiring highly specific skills, in order to be able to cope with the rapid developments of individual disciplines. Although I believe that this strategy will continue to be important, there is an increased demand for scientists to be able to put the pieces of specialist knowledge together in order to reveal the bigger picture.

This call for scientific synthesis not only addresses those who work in the applied world of resource management, but also those of us who address the basic 'blue skies' research questions that might reveal ground-breaking revelations.

Cross-boundary thinking requires a different approach to conceptualising problems, if not a change in academic culture. As an evolutionary biologist evaluating work done in previous decades fostering this mentality requires a

\section{S. Thatje $(\bowtie)$}

National Oceanography Centre, Southampton,

School of Ocean and Earth Science, University of Southampton,

European Way,

Southampton, SO14 3ZH, UK

e-mail: svth@noc.soton.ac.uk redefinition of scientific approaches from first principles. This is particularly poignant in a year in which we celebrate one of the greatest thinkers of all, Charles Darwin, who published 'On the Origin of Species' only 150 years ago. His groundbreaking work has changed our view of ourselves, the world we are living in, and even our society.

As a peer-reviewed international journal, NAWI will continue to advocate the development of multidisciplinary thinking. The academic focus of the NAWI has shifted under the influence of several Editors-in-Chief over time. From 2000-2009, Tatiana Czeschlik has led the journal to its current height in performance with an impact factor of 1.955 , and ranked $7^{\text {th }}$ in the Multidisciplinary Science Journal category (Thomson, 2007). Tatiana has recently decided to step-down from her responsibility as principal Editor-in-Chief, and since 1 February 2009, I have taken on the responsibility to lead the journal beyond its 100 years in existence. I am extremely grateful that Tatiana has agreed to lead me through the interim period, and as a continuous source of information and feedback. Our joint lead as Editors-in-Chief throughout 2009 will guarantee continuity whilst I implement my ambitions for further development of the journal.

I wish to conclude these editorial thoughts by focusing on some technical changes to the journal, most of which have already become day-to-day practice: NAWI is now accompanied by the subtitle-The Science of Naturegiving credit to the fact that English is undoubtedly the lead academic language at the present time. As a matter of further internationalising the journal, the editorial board is currently undergoing a major revamp by incorporating new members. This simply reflects that a few specialists cannot evaluate the whole scope of the natural sciences. A sound peer-review process thus demands a wide range of expertise to cope with the challenges involved in assessing the 
quality and significance of academic work. Expanding the editorial board also allows us to more easily deal with the steadily increasing number of manuscripts submitted to NAWI annually.

NAWI continues to publish a set of established paper types including a new type of contribution-Comments \& Replies - all of which are briefly defined as follows:

Original Articles - continue to remain the core of the journal and must present scientific results that are essentially new and that have not been published or are not being considered for publication elsewhere. The length of Original Articles should not exceed seven printed pages, which corresponds to 20-30 manuscript pages (double-spaced, 12-font, including everything from title page through the last figure). For the publication of unusually long methodological descriptions and data or figures that are not core to the publication, the publication of an online supplement is encouraged.

Reviews - are usually commissioned by the Editor or an Editorial Board member, but proposals for reviews are very welcome. NAWI now encourages the submission of a proposal rather than a full manuscript, which reduces efforts for both potential authors and editors. Proposals should not exceed 1,500 words in length and advocate for the significance and timeliness of the topic. Proposals should be sent to the Editor-in-Chief via e-mail. Reviews should cover a topic of current interest and present novel insights or conclusions for its interdisciplinary readership. The length should not exceed 85,000 characters (incl. blanks, but excluding Abstract, Legends and Tables). A review may have up to seven figures and/or tables.

Short Communications - are short papers that present significant new observations. Short Communications may present results that are not sufficiently elaborated but provide compelling evidence for their potential significance. This paper format is not to promote minimal or otherwise insufficient data sets that do not justify an Original Article. The manuscript length of Short Communications should not exceed 15,000 characters including spaces from beginning of Introduction to end of References and may have up to three figures or tables.
Comments \& Replies-is a new paper section in NAWI. Comments \& Replies give corrections, new analysis, provide critique or stimulate a more general oversight on contributions previously published in the journal. Comments should not refer to articles published in NAWI older than one year from online first publication. Comments are usually not exceeding 600 words in length, with a maximum of 10 references. They should present a simple message that requires only one small figure or table if any. Comments do not function as a Corrigendum to an article. This article type does not include an Abstract, but the title page should contain a footnote, providing an explicit reference to the criticized article "This is a comment to Authors (Year) Title. Naturwissenschaften, Volume: pages or DOI". Upon receipt of a Comment the authors of Original Articles are given the opportunity to reply. A Reply is usually published alongside the comment. Both Comments \& Replies are subject to peer review, usually by the original referees and/or editors of the article to which they refer.

The success of a journal is undoubtedly dependant on the support of its peer-review college. I am grateful for the support that the journal has received over many years by several thousands of scientists around the globe. Time from submission to publication of one's work is very important in the development of academic careers. The competitiveness of a journal to attract the very best contributions is reliant upon a timely response from reviewers, which must be done without weakening the effectiveness of the peer review process itself. NAWI has recently strengthened peerreview times and feedback mechanisms in response to the demands by authors. NAWI invites the submission of the very best of work from across the natural sciences community that reflects cutting edge research of interdisciplinary nature.

Sven Thatje

Editor-in-Chief

Naturwissenschaften

Southampton, 4 March 2009 